\title{
A novel virtual hospital at home model during the coronavirus disease 2019 (COVID-19) pandemic
}

\author{
Patrick P. Ryan MD, MPH ${ }^{1,2}$ (1), Kellie L. Hawkins MD²,3, Stacey Altman RN4, Lisa Granatowski RN ${ }^{4}$, Bradley D. Shy MD ${ }^{2,5}$, \\ Jeremy Long MD, MPH ${ }^{1,2}$ and Rebecca Hanratty MD ${ }^{1,2}$ \\ ${ }^{1}$ Department of General Internal Medicine, Denver Health \& Hospital Authority, Denver, Colorado, ${ }^{2}$ University of Colorado School of Medicine, Aurora, Colorado, \\ ${ }^{3}$ Division of Infectious Disease, Department of Medicine, Denver Health \& Hospital Authority, Denver, Colorado, ${ }^{4}$ Ambulatory Care Services, Community Health Services, \\ Denver Health \& Hospital Authority, Denver, Colorado and ${ }^{5}$ Department of Emergency Medicine, Denver Health \& Hospital Authority, Denver, Colorado
}

The COVID-19 pandemic has prompted healthcare systems to rapidly adapt healthcare delivery to accommodate a novel infectious disease while considering infection control practices, hospital capacity, and continued management of other medical conditions. Additionally, the COVID-19 pandemic has disproportionately affected minority communities and those suffering from lower socioeconomic status in the United States; populations that already face worse outcomes in other chronic medical conditions such as hypertension, coronary artery disease, and diabetes. ${ }^{1-3}$

To prepare for possible surge capacity, we built a virtual hospital at home $(\mathrm{VHH})$ to manage patients if the hospital could no longer accommodate new admissions. However, in response to early experiences with patients discharged from the emergency department (ED) who later decompensated, we pivoted to provide care to high-risk patients who did not meet the admission criteria.

In this report, we describe the development and implementation of a VHH program at an urban safety-net healthcare system to provide remote monitoring for high risk patients with COVID19 and the early outcomes associated with this program.

\section{Methods}

\section{Objectives}

The VHH was implemented to provide a safe home monitoring program for COVID-19 patients that did not meet admission criteria to provide an overflow contingency plan if our hospital reached capacity. Secondary goals included analyzing patient outcomes, and assessing the feasibility and acceptability of the $\mathrm{VHH}$ in a safety-net system.

\section{Setting and patient population}

Denver Health is an integrated, safety-net healthcare system. Most patients have government insurance (54\% Medicaid) or are uninsured $(18 \%)$. More than half of patients are members of racial/ethnic minority groups: $37 \%$ are Hispanic and $14 \%$ are black.

\section{Virtual hospital at home design}

Patients eligible for $\mathrm{VHH}$ monitoring include suspected or confirmed COVID-19 cases and at least 1 risk factor for disease complications but do not meet admission criteria (Fig. 1). ${ }^{1} \mathrm{VHH}$

Author for correspondence: Dr Patrick P. Ryan, E-mail: patrick.ryan@dhha.org Cite this article: Ryan PP, et al. (2021). A novel virtual hospital at home model during the coronavirus disease 2019 (COVID-19) pandemic. Infection Control \& Hospital Epidemiology, 42: 1140-1142, https://doi.org/10.1017/ice.2020.435 patients are provided a pulse oximeter and an automated blood pressure cuff to complete home monitoring assessments. $\mathrm{VHH}$ patients receive 2 phone calls per day: one from an $\mathrm{RN}$ and another from a provider to assess for interval symptom progression. $\mathrm{VHH}$ providers represent outpatient attending physicians, resident physicians, and advanced practice providers (ie, nurse practitioners and physician assistants).

Patients demonstrating clinical deterioration are transferred to the nearest $\mathrm{ED}$ via a coordinated effort between the $\mathrm{VHH}$ program and local EMS. Patients remaining clinically stable and meeting discharge criteria from the $\mathrm{VHH}$ are scheduled with their primary care provider (PCP) or receive assistance to establish care if they do not have a PCP.

This project was approved by the institutional quality improvement review committee.

\section{Results}

In total, 233 patients were referred to $\mathrm{VHH}$ for home monitoring between April 3 and May 24, 2020. The mean patient age was 49 years, and the most common risk factors for decompensation were hypertension, obesity, and diabetes (Table 1).

The average duration of $\mathrm{VHH}$ monitoring was 4 days. Of enrolled patients, 190 were successfully discharged from the program, $31(13.3 \%)$ required an escalated level of care, and $11(35.4 \%)$ required admission (Table 1 ). The $\mathrm{VHH}$ was unable to contact 13 patients (5.6\%). Most patients were uninsured $(28.3 \%)$ or were covered by Colorado Medicaid (38.2\%).

\section{Discussion}

The VHH provided a safe and effective mechanism to remotely monitor a population that has been disproportionately affected by the COVID-19 pandemic. As 95\% of patients referred participated, it seemed to be well received by patients, and the program successfully managed most patients within their own homes. Additionally, home-monitoring equipment was well received by patients referred to the $\mathrm{VHH}$, and these tools helped care providers assess the need for care escalations.

The VHH also assisted in managing hospital capacities through 2 distinct routes. First, the VHH allowed ED providers a mechanism to refer patients to the home-monitoring program who may have otherwise been admitted to an observation inpatient stay, avoiding an influx of admissions for lower acuity patients. Second, the $\mathrm{VHH}$ provides hospitalists an outlet to safely discharge COVID-19 patients earlier, knowing that patients will have close outpatient oversight and guidance in their care.

(C) 2020 by The Society for Healthcare Epidemiology of America. All rights reserved. This is an Open Access article, distributed under the terms of the Creative Commons Attribution licence (http://creativecommons.org/licenses/by/4.0/), which permits unrestricted re-use, distribution, and reproduction in any medium, provided the original work is properly cited. 


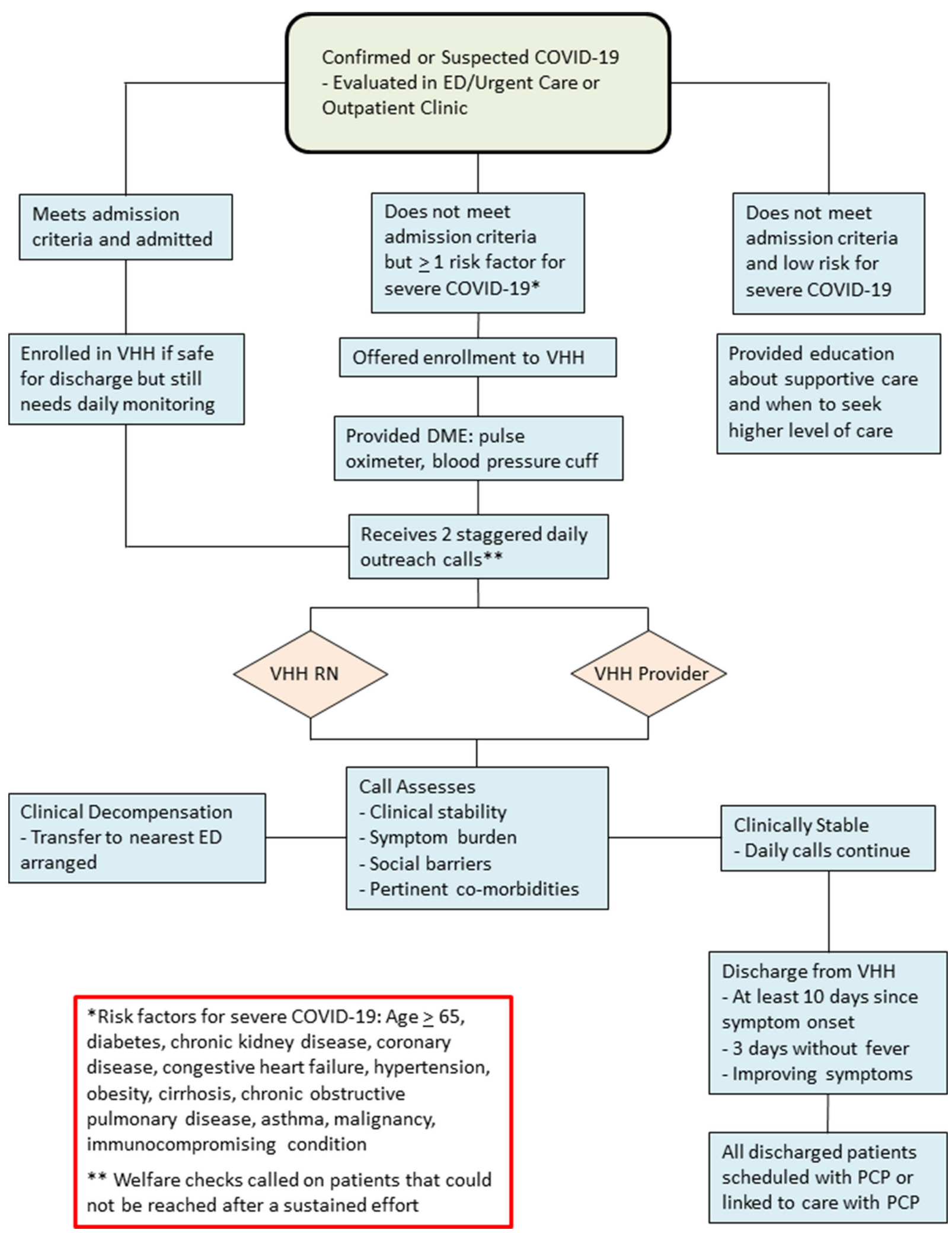

Fig. 1. Flow Diagram of Virtual Hospital at Home Design and Protocol.

Although prior hospital-at-home programs have used home visits as the care delivery mechanism, the VHH built upon these constructs exclusively through telehealth, while demonstrating program feasibility and safe patient care. ${ }^{4,5}$ By exclusively utilizing a telehealth approach, the VHH minimized the impact on inpatient PPE supplies during the COVID-19 pandemic.

Furthermore, this project demonstrates the acceptance of a novel telehealth program among a diverse patient population, most 
Table 1. Patient Demographics and Outcomes

\begin{tabular}{|c|c|}
\hline Characteristic & Overall No. (\%) \\
\hline Age, mean y (SD) & $49.2(14.7)$ \\
\hline$\geq 65$ & $34(14.6)$ \\
\hline Male & $108(46.4)$ \\
\hline Does not have PCP & $53(22.8)$ \\
\hline \multicolumn{2}{|l|}{ COVID-19 Status } \\
\hline Confirmed & $129(55.4)$ \\
\hline Negative & $72(30.9)$ \\
\hline Test Unavailable & $32(13.7)$ \\
\hline \multicolumn{2}{|l|}{ Race/Ethnicity } \\
\hline White & $55(23.6)$ \\
\hline Black & $22(9.4)$ \\
\hline Hispanic & $145(62.2)$ \\
\hline Other & $11(4.7)$ \\
\hline \multicolumn{2}{|l|}{ Comorbid conditions } \\
\hline Diabetes mellitis & $60(25.8)$ \\
\hline Coronary artery disease & $9(3.9)$ \\
\hline Chronic heart failure & $7(3)$ \\
\hline End-stage renal disease & $2(0.9)$ \\
\hline Hyoertrension & $142(60.9)$ \\
\hline Cirrhosis & $4(1.7)$ \\
\hline Asthma/COPD & $54(23.2)$ \\
\hline $\mathrm{BMI}>30$ & $96(41.2)$ \\
\hline Malignancy & $6(2.6)$ \\
\hline Immunosuppressed & $23(9.9)$ \\
\hline \multicolumn{2}{|l|}{ Referral source } \\
\hline Emergency department & $80(34.3)$ \\
\hline Urgent care & $57(24.5)$ \\
\hline Ambulatory testing & $54(23.2)$ \\
\hline Hospitalist service & $36(15.5)$ \\
\hline Other & $6(2.6)$ \\
\hline \multicolumn{2}{|l|}{ Preferred patient language } \\
\hline English & $131(56.2)$ \\
\hline Spanish & $94(40.3)$ \\
\hline Non-English/Non-Spanish & $8(3.4)$ \\
\hline \multicolumn{2}{|l|}{ VHH outcomes } \\
\hline Mean Duration of VHH Monitoring, d (SD) & $4.1(3.1)$ \\
\hline Discharged from $\mathrm{VHH}$ & $190(81.6)$ \\
\hline ED visit & $19(8.2)$ \\
\hline Hospital admission & $9(3.9)$ \\
\hline ICU admission & $2(0.9)$ \\
\hline Unable to contact & $13(5.6)$ \\
\hline
\end{tabular}

Table 1. (Continued)

\begin{tabular}{lc}
\hline Characteristic & Overall No. (\%) \\
\hline Insurance coverage & \\
\hline Commercial & $37(15.9)$ \\
\hline Medicaid & $89(38.2)$ \\
\hline Medicare & $34(14.6)$ \\
\hline Uninsured & $66(28.3)$ \\
\hline Unknown/Other & $7(3)$ \\
\hline
\end{tabular}

Note. SD, standard deviation; $\mathrm{PCP}$, primary care provider; COPD, chronic obstructive pulmonary disease; BMI, body mass index; $\mathrm{VHH}$, virtual hospital at home; ICU, intensive care unit.

of whom were ethnic minorities and were either uninsured or relied on some form of government insurance. As the COVID19 pandemic has disproportionately affected ethnic minority groups, more widespread implementation of telehealth home monitoring programs may even out this health disparity, but further study is needed to address this particular question.

Lastly, the VHH provides an opportunity to connect patients to longitudinal primary care. The VHH assisted unestablished patients to connect with enrollment services to implement medical coverage options, and it helped give patients direct access to scheduling in primary care clinics.

This evaluation has limitations. The program was designed to be a clinical intervention, not a prospective study, and our study cohort did not have a comparison group. Only patients with a functioning phone number could participate. The results are observational and may not be generalizable to other healthcare settings. Further study is necessary to understand differences in outcomes and the financial impact of $\mathrm{VHH}$ monitoring compared to observation hospitalizations for COVID-19 patients with similar risk profiles.

Acknowledgments. The authors would like to thank all of the Virtual Hospital at Home staff for their dedication to outstanding patient care.

Financial support. No financial support was provided relevant to this article.

Conflicts of interest. All authors report no conflicts of interest relevant to this article.

\section{References}

1. Garg S, Kim L, Whitaker M, et al. Hospitalization rates and characteristics of patients hospitalized with laboratory-confirmed coronavirus disease 2019COVID-Net, 14 states, March 1-30, 2020. Morb Mortal Wkly Rep 2020;69:458-464.

2. Webb Hooper M, Napoles AM, Perez-Stable EJ. COVID-19 and racial/ethnic disparities. JAMA 2020;323:2466-2467.

3. Singh GK, Lin CC. Area deprivation and inequalities in health and health care outcomes. Ann Intern Med 2019. doi: 10.7326/M19-1510.

4. Levine DM, Ouchi K, Blanchfield B, et al. Hospital-level care at home for acutely ill adults: a pilot randomized controlled trial. J Gen Intern Med 2018;33:729-736.

5. Shepperd S, Iliffe S, Doll HA, et al. Admission avoidance hospital at home. Cochrane Database Syst Rev 2016;9:CD007491. 